\title{
GAMMA-RAYS FROM 3C279 AND \\ UNIVERSAL ENERGY SPECTRUM
}

\author{
YUKIO TOMOZAWA \\ Randall Laboratory of Physics \\ University of Michigan \\ Ann Arbor, MI 48109-1120
}

\begin{abstract}
It has been suggested that the energy spectrum from point sources such as galactic black hole candidates (GBHC) and active galactic nuclei (AGN) is universal on the average. Recent measurements of $\gamma$-rays from 3C279 by the EGRET detector of Compton Observatory (alias GRO) are discussed and compared with the source spectrum of cosmic rays.
\end{abstract}

The origin of cosmic rays remains an open question to this date despite extensive studies in the past three quarters of a century. There are two outstanding sources of energy output in the sky: supernovae and black holes such as quasars, AGN and GBHC. These are obvious candidates for high energy particle generation. Shock wave acceleration in supernovae has been the most popular mechanism for the source of cosmic rays. It has some nice features but also has some difficult problems. The former is the question of the chemical composition in particular, the overabundance of iron, while the latter includes the question of high energy components of cosmic rays. Besides, it is very difficult to prove this hypothesis since there is no way of tracing the origin of cosmic rays due to the presence of the galactic magnetic field.

An alternative hypothesis is to relate cosmic ray acceleration with AGN and GBHC. A distinctive feature of these models is that cosmic ray particles may be accelerated by gravitational forces ${ }^{[1,2]}$ in contrast to electromagnetic acceleration in supernova. If that is the case, particles are accelerated irrespective of their charges: Neutral particles are also accelerated in the same way as cosmic rays are.

From these considerations, we have suggested the following:

1. All particles emitted by point sources such as quasars, AGN and GBHC have a universal energy spectrum. The shape of the universal spectrum should be identical to that of cosmic rays at the source.

2. Gamma-rays from AGN and GBHC should have the same energy spectrum as that of the source spectrum of cosmic rays. 
3. There is a possibility of the existence of a knee energy for the gamma ray spectrum at which an abrupt change of the slope parameter occurs as in the cosmic ray energy spectrum. If this phenomenon were to be observed by high energy gamma ray telescopes, it would provide strong observational support for the model.

The similarity between the energy spectrum for gamma-rays from $3 \mathrm{C} 273$ and cosmic rays was observed earlier ${ }^{[1,2]}$ although the error bars ${ }^{[3]}$ of the former were too large to be conclusive. More accurate observations of gamma-rays from 3C279 have been provided by Compton Observatory (alias GRO, Gamma Ray Observatory). The energy spectrum obtained is ${ }^{[4]}$

$$
\frac{d I}{d E}=(2.7 \pm 0.3) \times 10^{-7} E^{-(2.02 \pm 0.07)} \mathrm{cm}^{-2} \mathrm{~s}^{-1} \mathrm{GeV}^{-1}
$$

for the energy range of $30 \mathrm{MeV}-5 \mathrm{GeV}$. The power index $\lambda$ of the energy spectrum $E^{-\lambda}$ for cosmic rays is 2.6 above $1 \mathrm{GeV}$. (Below $1 \mathrm{GeV}$, it suffers from the solar modulation.) The important question is what is the power index at the source. The most elaborate estimate based on spallation and chemical abundance of cosmic rays gives ${ }^{[s]}$

$$
\lambda \simeq 2.2 \pm 0.1
$$

The comparison of Eqs. (1) and (2) seems to suggest consistency with the prediction of universality, although a further improvement in the estimate for the initial power index is required.

In order to further test the validity of the universality it is desirable to observe the gamma-ray spectrum from many more AGN and GBHC sources. In particular, the observation of gamma rays from galactic point sources, i.e. GBHC would be very important.

Finally, observation of the knee energy in the gamma-ray energy spectrum by high energy gamma ray telescopes would have a decisive impact on the whole subject of the origin of cosmic rays.

\section{ACKNOWLEDGEMENTS}

It is a great pleasure to thank Carl Fichtel, David Thompson and Simon Swordy for useful discussions. Thanks are also due to David Williams for reading the manuscript. This work is supported in part by the U.S. Department of Energy. 


\section{REFERENCES}

1. Yukio Tomozawa, Universality of the Energy Spectrum from Point Sources, UM-TH-91-14 (1991).

2. Yukio Tomozawa, Universal Energy Spectrum from Point Sources, to be published in the proceedings of the GRO Workshop, September, 1991.

3. G.F. Bignami et al. Astr. Ap. $\underline{93}$ (1981) 71.

4. R.C. Hartman et al., Detection of High Energy Gamma Radiation from Quasar 3C279 by the EGRET Telescope, to be published in Ap. J.

5. S.P. Swordy et al., Ap. J. $\underline{349}$ (1990) 625; D. Müller et al., Ap. J. $\underline{374}$ (1991) 356; P. Meyer et al., ICRC (1991) OG6.1.11. 